\title{
Repercusión social del embarazo en adolescentes
}

\author{
Social impact of teenage pregnancy
}

DOI: $10.46932 / \mathrm{sfjdv2n3-032}$

Received in: May 1st, 2021

Accepted in: Jun 30th, 2021

\section{Solange Karina Quijije Segovia \\ MSc}

Facultad Ciencias de la Salud / Carrera de Enfermería, Universidad Estatal del Sur de Manabí

E-mail: solangekarinaquijije@ hotmail.es,gina_am26@hotmail.es

\begin{abstract}
RESUMEN
La investigación tuvo como objetivo analizar la realidad de la educación familiar y el embarazo a temprana edad en el país, el cual se considera como problema social y de salud pública siendo el grupo de mayor vulnerabilidad las adolescentes con deficiencia de conocimientos en educación sexual, el diagnóstico demostró que la mayoría de los casos tienen afectaciones en la salud de la madre y del hijo por nacer, ocasionando alteraciones en la calidad de vida de ellas, familia y sociedad, los padres a su vez no brindan la suficiente confianza para dialogar sobre estos temas transcendentes, buscando en personas ajenas al hogar información que puede ser errada. Se utilizó los siguientes métodos teóricos: inductivo-deductivo y descriptivo, y como métodos empíricos encuestas a padres, adolescentes y entrevistas a especialistas en la temática de investigación. La investigación se realizó en el centro de salud Jipijapa, con las adolescentes embarazadas controladas, edades que oscila entre 17 a 19 años representando el grupo de mayor frecuencia de atención y con 3 embarazadas entre 10 a 13 años de edad. Un dato importante de destacar es un elevado porcentaje de padres de familia que no mantienen un dialogo fluido con sus hijos. El aporte teórico del trabajo lo constituyó la fundamentación de un club de madres adolescentes y una escuela para padres surgiendo participación activa de ambos grupos como alternativa para dar solución al problema planteado.
\end{abstract}

Palabras claves: Curiosidad, desorientación, libertad, vulnerabilidad

\begin{abstract}
The research had as objective analize the reality of the familiar education and pregnancy to early age in the country, which consider as social problem and public health being the vulnerability main group the teenagers with deficiency of knowledges in sexual education, the diagnostic showed that mayority of the cases have affections in the health of the mother and son to born, causing changes in the quality of life of their family and society, the parents not give the sufficient confidence to dialogue about these trascendents topics, searching in people to home information that can wrong. Used the following theorical methods descriptive inductive deductive and as empirical method as surveys to parents and teenagers and interviews with specialists in the topic of researchs field. The research was conducted at health center Jipijapa, with pregnants teenagers controled, ages that ranging from 17 and 19 representing the mayor group of more frequent attention and 3 pregnants from 10 to 13 years old. An important point to note is a high percent of parents of famiy that not care a dialogue fluid with their sons. The theorical contribution from job constituted the foundametation of a club of pregnants mothers and a parents schcool emerging active participation of both groups as an alternative to give solution to established problem.
\end{abstract}

Keywords: Curiosity, desorientation, freedom, vulnerability. 


\section{INTRODUCCIÓN}

La adolescencia es el periodo en el cual el individuo inicia su capacidad reproductiva, cambios tanto en la parte física y psicológica como la emocional, definen su identidad, siendo la base para adquirir su propia personalidad, es el paso de la niñez a la pubertad y luego a la adultez y puede lograr consolidar su independencia socio-económica, se consideran tres etapas de la adolescencia: la temprana entre los 10 y 14 años, intermedia entre 15 y 17 años y tardía 17 y 19 años de edad. (1)

La adolescencia es la etapa donde se presentan grandes oportunidades pero también es la más difícil y vulnerable, donde hay que hacer énfasis sobre la educación sexual de cada uno de ellas ya que un embarazo a temprana edad va a conllevar que el futuro de ellas y su hijo no sea muy exitoso, sobre todo en la parte socioeconómico. (2) (3)

La pubertad es una época de vida muy complicada donde se dan muchos cambios tanto biológicos como psicológicos, es aquí donde nace la preocupación para muchos, puesto que el embarazo precoz sobrelleva a que los jóvenes asuman responsabilidades de gran magnitud a temprana edad. Esto se ha convertido en un problema social y de salud pública, es por esta razón que se debe de educar a los jóvenes acerca de todo lo que compete a sexualidad según indica. (4)

A nivel provincial, nacional y mundial en los últimos años se ha presentado un incremento de embarazos precoces debido a que el inicio de la actividad sexual de las jóvenes está siendo a muy temprana edad, quizás ocasionados por falta de comunicación por parte de la familia, las disfunciones familiares, la falta de recursos económicos, entre otros (5).

Los autores Paula León, Matías Minassian, refieren que el embarazo en adolescentes se ha convertido en un problema de salud pública, debido a que cada vez es más frecuente que las jóvenes sean madres de familia precozmente, ocasionando falta de oportunidades de superación personal para el entorno social. $(6,7)$

Para contribuir a la disminución del embarazo en las adolescentes se deben realizar fortalecimiento institucional de los servicios de salud, educación y protección social, en un marco de coordinación interinstitucionales e intersectorial con activa participación de la sociedad. (8)

Según investigaciones realizadas a los padres indican que es la etapa más difícil que enfrentan con sus hijos, pero para quienes la han vivido y lo han hecho de manera tranquila y responsable se puede interpretar como la fase más bonita de la vida, viviendo a plenitud la etapa del primer amor, la primera ilusión y hasta la primera relación sexual, necesitando mucha responsabilidad ya que el hecho de querer experimentar esa experiencia sexual no solo puede ocasionar un embarazo no deseado sino también contraer infecciones de transmisión sexual, que pueden perjudicar la vida no solo de la adolescente sino también de su hijo. (9) 
Siendo la familia el grupo integrador denominado primera institución social o núcleo familiar, debe servir como principal fundamento para el desarrollo de cada individuo en temas educacionales sobre todo en sexualidad, pero debe ser en base a conocimiento de sus padres, con un nivel de afecto y comprensión, deben brindar protección, con mensajes de orientación adecuados e implantar comunicación oportuna para crear lazos de confianza dentro del hogar convirtiéndose los padres en la guía de crecimiento de sus hijos en todo ámbito para que logren la madurez correcta y se conviertan en adultos íntegros y útiles para la sociedad refiere. (10)

El dialogo entre padres e hijos debe ser un elemento fundamental y eficaz para garantizar la salud sexual y reproductiva de los jóvenes y puedan tomar decisiones adecuadas, actuar con responsabilidad cuando se vuelvan sexualmente activos, los padres deben brindarles la confianza necesaria para que los jóvenes puedan intercambiar información con ellos sobre sentimientos, relaciones románticas y humanas con la sociedad que lo rodea, ya que la adolescencia es una etapa de aislamiento y poca comunicación. (11)

Podemos indicar que la historia de la educación familiar, desde su principio ha sido esclava de grandes cambios pero este sigue siendo la parte fundamental para alcanzar los logros o caídas en los individuos, la familia educadora es sobre todo, trasmisora de vida inculcadora de valores que rehabilitan el lienzo social infundidos desde el centro familiar para ser difundido a toda las personas". (12)

La educación dentro del núcleo familiar debe ser afectiva con buenos vínculos de comunicación para lograr transmitir información eficaz y oportuna a los jóvenes de manera que puedan ser orientados en todos los problemas sociales que se puedan ver involucrados por desconocimiento y así impedir que los adolescentes actúen de forma inapropiada tomando decisiones que pueden poner en riesgo su salud o su vida, es por esto que ellos precisan del apoyo y orientación familiar, de todas las personas que se encuentran en su entorno social inculcando los principios y valores siendo cualidades muy importantes para su comportamiento ante la sociedad. (13)

Antiguamente las familias de pueblos primitivos cuando no existía la formación académica para el desarrollo del conocimiento educaban a sus hijos desde tempranas edades dentro del hogar de manera estricta, reproduciendo las enseñanzas dictadas por sus padres, inculcando valores morales, adorando sus orígenes; a medida que ha transcurrido el tiempo las familias han adoptado nuevas estrategias de educación para sus primogénitos y estos los han ido transmitiendo y moldeando de acuerdo al paso del tiempo moderno para el correcto aprendizaje añade (Soliz 2021).

Según (Cortes, 2021) manifiesta que la familia es la principal institución encomendada a transmitir conocimientos, valores, actitudes, cuando nos referimos a la familia debemos especificar a qué tipo pertenecen: 
Familia nuclear: Está conformada por el padre la madre y sus hijos.

Familia extendida: Se compone de uno de los dos progenitores (mamá o papá) un posible compañero (a), los hijos que puedan tener y otros familiares que viven con ellos en la misma casa.

Familia mixta o reconstituida: Está formada por una persona viuda o divorciada, con o sin hijos, que contrae de nuevo matrimonio la cual ante pudo haber estado casada o no, y puede tener o no hijos.

Familia binuclear: Es una familia que por causa del divorcio está dividida en dos, es decir consta de dos familias, una encabezada por el padre y otra por la madre.

Familia comunal: Es aquella conformada por un grupo de personas que viven juntas y comparten diversos aspectos de su vida. (4)

La pedagogía en valores debe estar presente en todo el proceso formativo, la intervención demanda gran participación de los padres y los hijos para realizar las actividades participativas y comunicativas, lo que implica una mejor organización en el campo familiar y escolar, los valores morales son muy importantes ya que deben experimentarse en el núcleo de la educación familiar mismo que hace referencia a valores en actitudes, conocimientos y hábitos en nuestro medio, la familia no puede olvidar que es en el hogar donde los hijos reciben las primeras caricias, las primeras enseñanzas y su comportamiento inicial así como los elementos éticos que deben regir a la persona a través de su vida en el seno de la colectividad familiar como indica. (14)

La educación que tengan los padres de los y las adolescentes es un instrumento muy importante, ya que de acuerdo a su instrucción académica es más fácil tener una comunicación efectiva con sus hijos en sentido de las conductas y comportamientos no saludables, sobre todo hablar de prevención en cuanto a embarazos y patologías así mismo a prender a través de la educación nuevas ideas que le ayuden a cumplir muy bien su rol de padres pedagógicos, realzando los conocimiento y animando la acción razonable de la sexualidad para que estos influyan a favor de cambios sociales, ambientales beneficiosos y saludables en los adolescentes refiere. (15)

El objeto de la investigación es el embarazo, el cual es causado probablemente por la falta de comunicación con sus padres, buscando información errada con amigas de su misma edad, vecinas, hermanas y otros, muchas veces sufren discriminación y vergüenza social, pero lo más terrible es la muerte de adolescentes y sus hijos, por las múltiples complicaciones que se presentan durante el mismo, por lo tanto la tarea de los padres será dar educación sobre temas de sexualidad sin tabú, brindando toda la confianza que las chicas necesitan a esta edad.

El campo de la investigación es Psico- Educativo, ya que la maternidad a temprana edad se está convirtiendo en un problema de salud pública a nivel provincial, nacional y mundial, siendo además una de las principales causas de morbi-mortalidad materno-infantil, debido a la falta de educación y de 
información sobre temas de sexualidad y métodos anticonceptivos, a pesar que hay programas educativos pero ellas las interpretan de manera inadecuada.

El objetivo de la investigación, es determinar la influencia de la educación en la familia en el embarazo de las adolescentes, constituyendo como valor fundamental la educación sexual, de manera que los jóvenes puedan tener orientación y garantizar una sexualidad responsable y satisfactoria.

En relación con el objetivo planteado en la investigación se precisaron las siguientes tareas científicas:

1. Analizar desde el punto de vista histórico la educación en la familia y el embarazo en las adolescentes.

2. Valorar los fundamentos teóricos sobre la deficiencia educativa familiar y su influencia en el embarazo en las adolescentes que acuden al centro de salud jipijapa.

3. Determinar el grado de educación que tienen las adolescentes sobre temas de sexualidad, mediante las encuestas realizadas.

La propuesta consiste en crear un club de madres adolescentes que se realizan los controles prenatales en el centro de salud jipijapa, en el cual se van a brindar orientación mediante la realización de actividades pedagógicas sobre embarazo, lactancia, alimentación, cuidados pre y post natales e incentivar a la planificación familiar, además se implementó una escuela para padres donde se plateo temas de educación sexual y como orientar a los adolescentes en estos temas.

La presente investigación se efectuó en el centro de salud jipijapa, la población tomada en cuenta está constituida por 79 adolescentes embarazadas entre las edades de 13 a 19 años de edad, en la que se eligió una muestra de 79 adolescentes embarazadas. El muestreo será aleatorio simple.

Para dar desempeño a las tareas científicas, se utilizaron los métodos teóricos y empíricos. En lo que concierne a los métodos teóricos es el método inductivo-deductivo y método descriptivo.

\section{MÉTODOS}

Se aplicó el método inductivo-deductivo, el cual se empleara para demostrar el análisis general de la educación en la familia y el embarazo en las adolescentes, con el propósito de conocer sus causas, consecuencias y se explicara la información difundida de esta investigación, implantando características determinadas del embarazo en los adolescentes que es de valiosa importancia en la estructuración de los resultados. Otro método empleado fue el descriptivo, el cual se aplicó en el proceso de la investigación especialmente para obtener datos estadísticos y características de la población, ya que están involucrados con los fenómenos que ocurren naturalmente como el déficit de educación padre e hijos. 
Figura 1

Se aplicó técnicas como las encuestas dirigidas a las adolescentes y padres de familia y entrevistas realizadas a los especialistas que laboran en el centro de salud jipijapa, para obtener la información sobre el problema planteado en la investigación.

\section{1 -A FIGURAS}

DISTRIBUCIÓN POR EDADES DE LAS ADOLESCENTES EMBARAZADAS Y CONTROLADAS QUE ACUDEN AL CENTRO

DE SALUD JIPIJAPA DE ENERO A MARZO DEL 2015

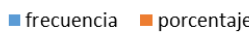

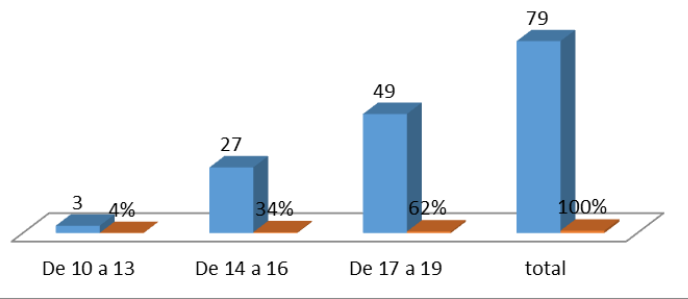

Figura 2

DIALOGA SOBRE TEMAS DE SEXUALIDAD CON SUS PADRES

frecuencia $\square$ porcentaje

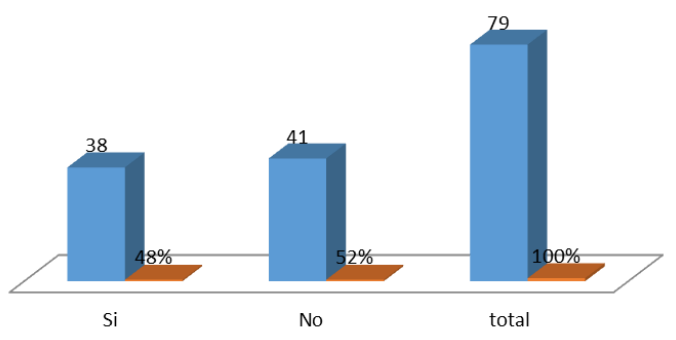

Figura 3

CONOCE LAS CONSECUENCIAS QUE IMPLICA TENER UN EMBARAZO A TEMPRANA EDAD

= frecuencia $=$ porcentaje

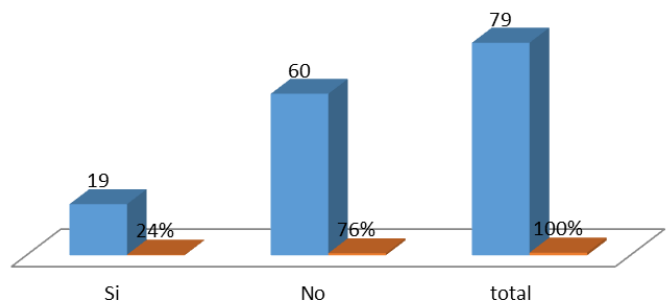

total 
Figura 4

ANTES DE QUEDAR EMBARAZADA USTED EMPLEABA MÉTODOS ANTICONCEPTIVOS

$\square$ frecuencia $\square$ porcentaje

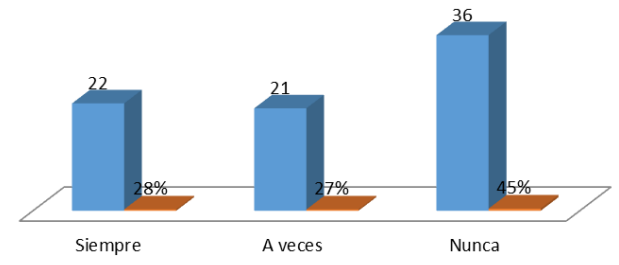

Figura 5

COMO ERA LA COMUNICACIÓN CON SUS PADRES ANTES DE QUEDAR EMBARAZADAS

a frecuencia porcentaje

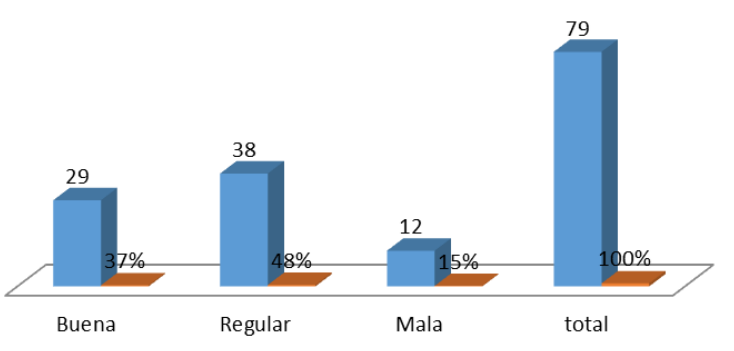

Figura 6

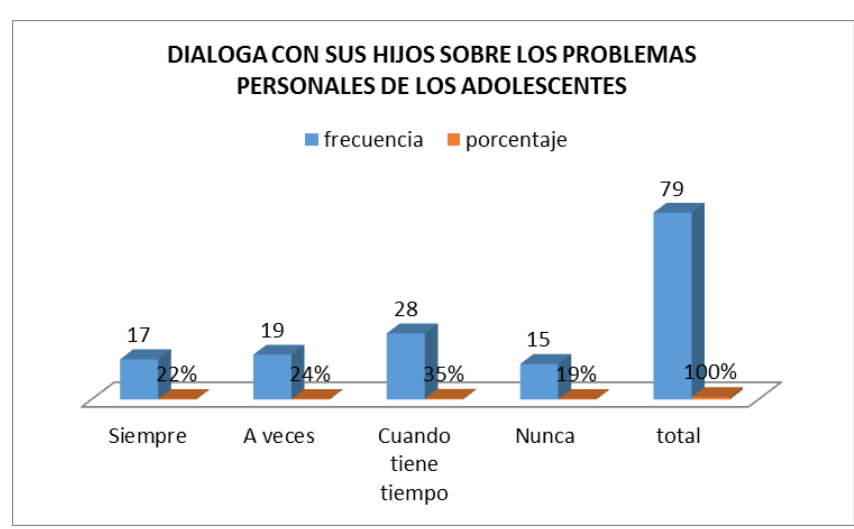

Figura 7

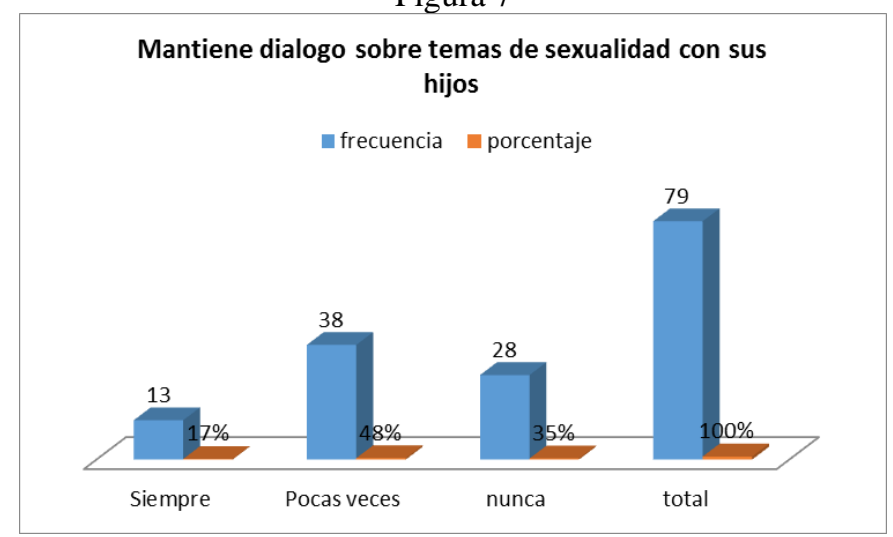




\section{Tabla I}

Distribución por edades de las adolescentes embarazadas y controladas que acuden al centro de salud jipijapa de enero a marzo del 2015.

\section{ALTERNATIVA FRECUENCIA PORCENTAJE}

\begin{tabular}{|ccc|}
\hline De 10 a 13 & 3 & $4 \%$ \\
\hline De 14 a 16 & 27 & $34 \%$ \\
De 17 a 19 & 49 & $62 \%$ \\
TOTAL & $\mathbf{7 9}$ & $\mathbf{1 0 0 \%}$ \\
\hline
\end{tabular}

Tabla II

Dialoga sobre temas de sexualidad con sus padres

\begin{tabular}{ccc}
\hline ALTERNATIVA & FRECUENCIA & PORCENTAJE \\
Si & 38 & $48 \%$ \\
No & 41 & $52 \%$ \\
TOTAL & $\mathbf{7 9}$ & $\mathbf{1 0 0 \%}$ \\
\hline
\end{tabular}

Tabla III

Conoce las consecuencias que implica tener un embarazo a temprana edad

\begin{tabular}{ccc}
\hline ALTERNATIVA & FRECUENCIA & PORCENTAJE \\
Si & 19 & $24 \%$ \\
No & 60 & $76 \%$ \\
TOTAL & $\mathbf{7 9}$ & $\mathbf{1 0 0 \%}$ \\
\hline
\end{tabular}

Tabla IV

Antes de quedar embarazada usted empleaba métodos anticonceptivos

\begin{tabular}{|ccc|}
\hline ALTERNATIVA & FRECUENCIA & PORCENTAJE \\
Siempre & 22 & $28 \%$ \\
A veces & 21 & $27 \%$ \\
Nunca & 36 & $45 \%$ \\
\hline
\end{tabular}




\section{Tabla V}

Como era la comunicación con sus padres antes de quedar embarazada

\begin{tabular}{|ccc|}
\hline ALTERNATIVA & FRECUENCIA & PORCENTAJE \\
\hline Buena & 29 & $37 \%$ \\
\hline Regular & 38 & $48 \%$ \\
\hline Mala & 12 & $15 \%$ \\
\hline TOTAL & $\mathbf{7 9}$ & $\mathbf{1 0 0 \%}$ \\
\hline
\end{tabular}

Tabla VI

Dialoga con sus hijos sobre los problemas personales de los adolescentes

\begin{tabular}{|ccc|}
\hline ALTERNATIVA & FRECUENCIA & PORCENTAJE \\
\hline Siempre & 17 & $22 \%$ \\
A veces & 19 & $24 \%$ \\
\hline $\begin{array}{c}\text { Cuando tiene } \\
\text { tiempo }\end{array}$ & 28 & $35 \%$ \\
Nunca & 15 & $19 \%$ \\
\hline TOTAL & $\mathbf{7 9}$ & $\mathbf{1 0 0 \%}$ \\
\hline
\end{tabular}

Tabla VII

Mantiene dialogo sobre temas de sexualidad con sus hijos

\begin{tabular}{ccc}
\hline ALTERNATIVA & FRECUENCIA & PORCENTAJE \\
\hline Siempre & 13 & $17 \%$ \\
Pocas veces & 38 & $48 \%$ \\
\hline NUNCA & 28 & $35 \%$ \\
\hline TOTAL & $\mathbf{7 9}$ & $\mathbf{1 0 0 \%}$ \\
\hline
\end{tabular}

\subsection{RECOMENDACIONES}

De acuerdo con el estudio realizado se sugiere que siendo el embarazo en adolescentes un problema social y de salud pública con el grupo de mayor vulnerabilidad, con deficiencia de conocimientos en educación sexual, se debe de incrementar más la prevención, por parte de los padres de familia siendo el primer lugar de formación de valores, en los colegios y lo más importante el gobierno de turno, poner mayor énfasis en esta problemática. 
Brindar capacitaciones frecuentes como parte de una campaña para evitar más jóvenes embarazadas porque no solo sufren ellas como adolescentes sino también sus hijos por falta de economía para sustentarlos, inexperiencia para los cuidados de los niños.

A los padres de familia incentivar al dialogo en temas de sexualidad con sus hijos sin ningún tabú, para que los jóvenes aprendan a respetar su cuerpo, brindarles confianza.

Que se implementen escuelas para padres donde allá la participación activa de ellos y sus hijos independientemente del sexo (hombre, mujer) para que se motive laso de unión familiar y colaboración en el hogar.

\section{ANÁLISIS DE RESULTADOS}

Según los datos obtenidos del "Centro de Salud Jipijapa" pudimos determinar que el grupo de edad de las adolescentes controladas, oscila entre 17 a 19 años que representa el grupo de mayor frecuencia de atención con un 62\%, seguido de las jóvenes en edades comprendidas entre 15 a 16 años con $34 \%$ y de 10 a 13 años con un $4 \%$ con un numero de 3 embarazadas. Por lo que se puede concluir la mayoría de las embarazadas adolescentes se dio en las de etapa tardía entre 17 a 19 años de edad.

De acuerdo al dialogo sobre los temas de sexualidad dentro del hogar entre padres e hijos en un $52 \%$ refieren no tener ese tipo de conversación con sus progenitores, un $48 \%$ si recibe ese tipo de enseñanza por parte de los padres, estos resultados nos dan a conocer la calidad de educación e información que trasmiten los padres a los hijos para orientarlos sobre la salud sexual y reproductiva lo que disminuye los embarazos a temprana edad en los adolescentes.

En la encuesta aplicada pudimos observar que las adolescentes embarazadas atendidas en el centro de salud jipijapa no tienen conocimientos sobre las consecuencias que implica tener un embarazo a temprana edad con un $76 \%$, y el $24 \%$ si tiene conocimiento sobre las consecuencias, lo que demuestra que las adolescentes embarazadas en su mayor parte no conocen sobre las complicaciones que conlleva tener un embarazo precoz, sin embargo la minoría tienen desconocimientos de esta etapa del embarazo.

Según la encuesta empleada nos indica que, el $45 \%$ de las adolescentes embarazadas atendidas en el centro de salud jipijapa nunca utilizo algún método anticonceptivo, el 28\% siempre utilizo algún método de anticoncepción, y el 27\% a veces cuando mantiene relaciones sexuales emplea algún método anticonceptivo, esto pone en evidencia la realidad de nuestra provincia en donde los jóvenes no utilizan algún método de anticoncepción, sin tomar en cuenta la responsabilidad que esta situación conlleva. 
En cuanto a la comunicación en el núcleo familiar se puede observar que el $48 \%$ es regular, un $37 \%$ es buena, y el $15 \%$ es mala, por lo que podemos deducir que las familias de las adolescentes embarazadas no tienen la confianza suficiente para entablar diálogo sobre los problemas de la adolescencia y el comportamiento así como también brindar apoyo moral y comprensión a los hijos.

En las encuestas realizadas a los padres podemos evidenciar que muchos no le dan el espacio suficiente a los jóvenes para, conversar sobre los problemas de la pubertad lo que se representa un 35\%, en un $24 \%$ a veces suelen conversar padre e hijos, con un $22 \%$ siempre hablan con sus hijos y finalmente un $19 \%$ de los casos nunca habla con los hijos, pensamos que es de gran importancia investigar la relación intrafamiliar de las adolescentes porque se observa mayor porcentaje de embarazo en precoces donde no existe comunicación adecuada entre padres e hijos.

Con respecto al dialogo sobre temas de sexualidad entre padres e hijos según los datos obtenidos podemos deducir que el $48 \%$ de los padres no mantienen dialogo sobre temas de sexualidad con sus hijos, en un 35\% nunca lo hace, y el $17 \%$ lo realiza muchas veces para que los jóvenes tengan la orientación apropiada, se puede observar que los padres no tienen conversación sobre estos temas por falta de conocimientos y desconfianza.

\section{CONCLUSIONES}

La principal prevención es mediante la educación de los padres la cual debe ser impartida desde los hogares, ellos primero deben informarse para poder hablar con lenguaje apropiado respecto a los órganos reproductores tanto femeninos como masculinos, es necesario saber cómo manejar en los jóvenes la sexualidad que desean lograr.

Enseñarles a respetar su cuerpo y que sepan tomar decisiones apropiadas cuando tengan una pareja sentimental por lo que empieza a despertar el deseo sexual donde nace la conocida frase "la prueba de amor", ante esta situación es necesario que los adolescentes tengan información sobre las relaciones sexuales, el uso de los anticonceptivos y conocer sobre las diferentes infecciones de trasmisión sexual y la manera de evitar un embarazo no deseado así mismo los miembros de la familia deben promover el cultivo de valores dentro del hogar.

La enseñanza sobre sexualidad y planificación familiar debe ser transmitida por personal capacitado que le brinde información adecuada sobre el uso de los métodos anticonceptivos y las relaciones sexuales así como el cuidado y tratamiento de las enfermedades de trasmisión sexual, conformando un club de adolescentes en el cual puedan ejecutar dinamismos y prácticas formativas para las y los jóvenes, así mismo brindar asesoramiento sobre sus derechos sexuales como, cuando y cuantos hijos desean tener en las circunstancias adecuadas y evitar los embarazos no deseados, se inculco a los 
padres de familia la importancia de ejecutar dialogo con los jóvenes de manera activa, afectiva y participativa, brindando tiempo de calidad no de cantidad, apoyándoles en sus tareas diarias, con lo cual los adolescentes se sientan en un ambiente de confianza y seguridad. 


\section{BIBLIOGRAFÍA}

1. Loredo A, Vasgas C, Casas M, González C, Gutiérrez LC. Embarazo adolescente: sus causas y repercusiones en la diada. Rev Med Inst Mex Seguro Soc. 2017; 55(2): p. 223-229.

2. Ramírez N, Córtez BM, Ruiz TA, Pérez LD, Buendía HA, et at. Pregnancy in teenagers with heart disease. Arch Cardiol Mex. 2020; 90(1): p. 81-85.

3. Molina A, Pena A, Días C. Condicionantes y consecuencias sociales del embarazo en la adolescencia. Revista Cubana de Obstetricia y Ginecología. 2019; 45(2): p. 1-21.

4. Cortez M, Santos A, Torres M, Teixeira L, Alves S. Análise das complicações clínico-obstétricas em gestantes adolescentes segundo a Classificação de Robson. Rev. enferm. UERJ. 2021; 29.

5. Moon TD, Okoth V, Joseth S, Opiyo E, et,at. Determinants of modern contraceptive prevalence and unplanned pregnancies in Migori County, Kenya: results of a cross-sectional household survey. Afr J Reprod Health. 2021; 25(1): p. 29-40.

6. Leon P, Minassian M. Embarazo adolescente. Revista Pediatría Electrónica. 2008;: p. 7-14.

7. Alban v. programa de atencion integral y diferenciada de los adolescentes en relacion a los conocimientos. guaranda.

8. Fahme S, Sieverding M. Sexual and reproductive health of adolescent Syrian refugee girls in Lebanon: a qualitative study of healthcare provider and educator perspectives. Reprod Health. 2021; 18(1): p. 113.

9. Morales N, Quintana L. Embarazo en adolescente: Problema de salud pública o de intervención social. Culcyt//Intervención Social. 2013;: p. 56.

10. Lilanos Nación M, Bejarano Soto D. Embarazo en adolescentes y sus complicaciones materno fetales en el Hospital materno infantil Carlos Showing Ferrari. Tesis, Universidad Nacional Hermilio Valdizán. 2017.

11. Aburto AM, Escamilla SR, Díaz OC, Fajardo DG, Urrutia AM, et,at. Sexual health educational intervention in medical students. Gac Med Mex. 2020; 156(2): p. 164-170.

12. Soliz Ordoñez L. Validación de instrumento para evaluar la prevalencia y consecuencias de los embarazos en la adolescencia del área de influencia del centro de salud macuma zona indígene shuar. Mas vita. 2021; 3(2): p. 30-39.

13. Ávalos DS , Recalde F, Cristaldo C, Puma AC, Carbonell LA, López P. Estrategia de unidades de salud familiar: su impacto en la tasa de embarazo en adolescentes en Paraguay. Rev Panam Salud Publica. 2018; 30: p. 42-59.

14. Álavrez J, Blanco A, Torres M, Guilarte O. Educational program for female teenagers. $\mathrm{ccm}$ [Internet]. 2018; 22(4): p. 559-700.

15. Venegas M, Valles N. Factores de riesgo que inciden en el embarazo adolescente desde la perspectiva de estudiantes embarazadas. Rev Pediatr Aten Primaria [Internet]. 2019; 21(83): p. 109-119. 


\section{APÉNDICES}

El trabajo realizado tiene mucha similitud con la del autor Montalvo González, trabajo realizado en Veracruz y universidad autónoma de la ciudad Juarez, ya que dentro de los factores que influyen en el embarazo de las adolescentes se debe a la falta de información, desorientación, mala comunicación, por parte de los padres, ocasionando en los adolescentes que busquen información errada en otras personas, que no se sientan en confianza con sus padres sino que allá temor al buscar en ellos el diálogo, comprensión y sobre todo confianza que debe ser lo fundamental para los adolescentes. Se logró con el trabajo ejecutar el club de madres adolescentes, se les brindo información de planificación familiar, enfermedades de transmisión sexual, y sobre todo el cuidado que deben tener para con su hijo, además se formó la escuela para padres realizando participación activa.

En base a los resultados de las encuestas realizadas a las adolescentes y padres de familia así como la entrevista aplicada a los expertos en la temática con respecto a la problemática se determinó que el grupo de mayor vulnerabilidad son las adolescentes, poniendo en riesgo la salud tanto de ellas como la de su hijo. Otros aspectos que se analizaron es la falta de comunicación entre padres e hijos con relación a los problemas de la pubertad, sobre la sexualidad y modo de prevención de embarazos no deseados así como también las diferentes patologías de trasmisión sexual a las que se exponen al realizar actividades sexuales sin protección poniendo en peligro su salud, además se determinó que la confianza entre padres e hijos no es tan buena al hablar sobre temas de sexualidad, ya que los padres creen que al dialogar sobre estos temas no están educando sino más bien incentivándolos a las relaciones sexuales prematuras. En cuanto a la entrevista los especialistas refieren, que los jóvenes tienen desconocimientos sobre la sexualidad, así como mala información sobre los métodos de planificación familiar y sobre todo un gran descuido de los padres dentro del hogar ya que muchos se dedican a las labores diarias, lo que incrementa el desconocimiento y con ellos los embarazos cada vez más temprano en las adolescentes. 